\title{
A New Low Cost Biosorbent for a Cationic Dye Treatment
}

\author{
Belbahloul Mounir, Msaad Asmaa, Beakou Buscotin, Houssaini Mohammed, Amine, \\ Zouhri Abdeljalil, Anouar Abdellah
}

Chemistry and Environment Laboratory, Faculty of Science and Technology, University Hassan 1, BP 577, Settat Morroco

\begin{abstract}
The aim of our study consists to investigate the adsorption of Methylene Blue from aqueous solution by a new biosorbent prepared from Papaya seed. Adsorption behavior of the cationic dye was analyzed by variation of solution $\mathrm{pH}$, contact time, adsorbent dose, and temperature. Adsorption isotherms were studied according to the Langmuir and Freundlich Model, and adsorption kinetics according to pseudo first and second order. Results show that the maximum adsorption is obtained at ambient temperature with the yield of $98.82 \%$ and was reached in first 20min ( $\mathrm{pH}=10$, adsorbent dose of $100 \mathrm{mg}$ in $50 \mathrm{~mL}$ ). The Langmuir isotherm shows a correlation coefficient of $99.4 \%$ higher than 95.4\%obtained for Freundlich model and the adsorption kinetic model follow a pseudo-second order with a maximum adsorption capacity of $52.28 \mathrm{mg} / \mathrm{g}$.
\end{abstract}

Keywords-Methylene Blue, adsorption, Papaya seed, adsorption isotherm and adsorption kinetic.

\section{INTRODUCTION}

Water pollution has been generated an enormous fund input and raised worldwide concern[1]. Textile wastewater, generally, contains a big amount of pollutants materials like colored materials or dyes, organic compounds and heavy metals ions.These materials can affect the physicochemical and the biological properties of sea, drinking water and globally the ecosystem. In addition to the undesirable colors of textile effluents, some dyes may degrade to produce carcinogens and toxic products [2]. Furthermore, the colored effluents reduce light penetration and potentially prevent photosynthesis. Dyes even in very low concentrations affect the aquatic life and food chain. In the recent years, researchers are indulging their interest in wastewater treatment by various processes such as precipitation, ion exchange, reverse osmosis and adsorption [3]. The adsorption of colored solution has been the main point of numerous researchesas the effective process because it's easy to do, produce sludge without chemical products like conventional wastewater treatment, selective and cost-effective[3].In this work, we used a new biosorbent, to treat the Methylene Blue (MB) by the batch adsorption system; the work consisted on the study of the effect of different factors which influenced the adsorption process as: $\mathrm{pH}$, adsorbent dose, contact time and temperature, to deduce the adsorption thermodynamic and kinetic behavior process.

\section{MATERIALS AND METHODS}

\subsection{Material and reagents:}

Different laboratories materials are used in this work like UV visible spectroscopy (HACH LANGE DR 6000) for determination of $\mathrm{MB}$ concentration at a wave number of $662 \mathrm{~nm}$, multi-parameter Consort C 3040 to adjust the $\mathrm{pH}$ values by $\mathrm{HCl}(0.1 \mathrm{~N})$ orNaOH $(0.1 \mathrm{~N})$ and multi agitator.Methylene Blue(MB) $\left(\mathrm{C}_{16} \mathrm{H}_{18} \mathrm{ClN}_{3} \mathrm{~S}\right.$ see Fig.1), obtained from Sigma Aldrich, with a molecular weight of $319.85 \mathrm{~g} / \mathrm{mol}$. Solutions were prepared by dilution with distillated water of the stock solution of MB with the initial concentration of $1000 \mathrm{ppm}$ to reach the desired concentration.<smiles>CN(C)c1ccc2nc3ccc(N(C)C)cc3[s+]c2c1</smiles>

Fig.1: Methylene Blue structure

\subsection{Characterization of the biosorbent:}

Papaya seedare very abundant in Morocco and not valorized for any use were collected from a manufacturing process in the location of Settat- Casablanca, Morocco, washed several times to eliminate the impurities, and thencrushed to obtain a powder used as a new biosorbent. Our product was used in wastewater treatment without any chemical or physical activation treatment. The physicochemical properties of our biosorbent were determined by Langmuir and Freundlich model.

\subsection{Adsorption experiments:}

The adsorption experiments of $\mathrm{MB}$ on the biosorbent were reached after $2 \mathrm{~h}$ of stirring at ambient temperature and 
agitation speed of $300 \mathrm{rpm}$. The suspensions were collected then centrifuged and the MB equilibrium concentrations were determined at $662 \mathrm{~nm}$. The yield of adsorption and the amount of $\mathrm{MB}$ adsorbed at equilibrium noted $\mathrm{R}$ in $(\%)$ and $\mathrm{q}_{\mathrm{e}}$ in $\mathrm{mg} \cdot \mathrm{g}^{-1}$, respectively, were calculated by the following equations (Eq.1 and Eq.2):

$$
\mathbf{R}(\%)=\frac{\mathrm{C}_{0-\mathrm{C}_{\mathrm{t}}}}{\mathrm{C}_{0}} .100 \quad(1) \mathbf{q}_{\mathrm{e}}=\frac{\left(\mathrm{C}_{0}-\mathrm{C}_{\mathrm{t}}\right) \mathrm{v}}{\mathrm{m}}(2)
$$

Where $\mathrm{C}_{0}$ is the initial dye concentration $\left(\mathrm{mg} \cdot \mathrm{L}^{-1}\right), \mathrm{C}_{\mathrm{t}}$ is the equilibrium dye concentration $\left(\mathrm{mg} \cdot \mathrm{L}^{-1}\right), \mathrm{V}$ is the volume of the solution and $\mathrm{m}$ is the mass of the adsorbent (g).

\subsubsection{Kinetic study:}

Adsorption kinetic experiments were carried out using batch model. All of the dye solution was prepared with distilled water. Kinetic experiments were carried out by agitating $100 \mathrm{ml}$ of solution of a constant dye concentration with $60 \mathrm{mg}$ of $\mathrm{MB}$ at a constant agitation speed, ambient temperature and $\mathrm{pH}=2$. Agitation was made from 5 to $120 \mathrm{~min}$. The experimental data will fitted by the pseudo-first-order and pseudo-second-order equation [4]

$$
\begin{aligned}
& \operatorname{Ln}\left(\mathrm{q}_{\mathrm{e}}-\mathrm{q}_{\mathrm{t}}\right)=\ln \mathrm{q}_{\mathrm{e}}-\mathrm{k}_{1} \mathrm{t} \\
& \frac{\mathrm{t}}{\mathbf{q}_{t}}=\frac{\mathbf{1}}{\mathrm{K}_{2} q_{e}^{2}}+\frac{\mathrm{t}}{\mathrm{q}_{\mathrm{e}}}
\end{aligned}
$$

Where:

$\mathrm{K}_{1}$ : the rate constant of the pseudo-second order model $\left(\min ^{-1}\right) ; \mathrm{q}_{\mathrm{t}}$ and $\mathrm{q}_{\mathrm{e}}$ are the amounts of dye adsorbed on biosorbent in $\mathrm{mg} / \mathrm{g}$ at time $\mathrm{t}$ and in the equilibrium respectively;

$\mathrm{k}_{2}$ is the pseudo-second order kinetic model rate constant in $\mathrm{g} / \mathrm{mg}$. $\min$

\subsubsection{Isotherm study:}

Different isotherm models provide us several information about the adsorption mechanism, the surface properties of the sorbent and the affinities between the sorbent and sorbate. In this work, two of the most used isotherms namely Langmuir and Freundlich, were used to fit the equilibrium experimental data of MBadsorption into our biosorbent. Langmuir theory assumes the existence of finite number of identical sites homogeneously distributed over the adsorbent surface, the linear form of this model is represented in the Eq.(5):

Where:

$$
\frac{\mathrm{C}_{\mathrm{e}}}{\mathrm{q}_{\mathrm{e}}}=\frac{1}{\mathrm{q}_{\max } \mathrm{K}_{\mathrm{L}}}+\frac{\mathrm{C}_{\mathrm{e}}}{\mathrm{q}_{\max }}(5)
$$

$\mathrm{q}_{\mathrm{e}}$ : the equilibrium dye concentration on the adsorbent (mg. $\left.\mathrm{g}^{-1}\right) ; \mathrm{C}_{\mathrm{e}}$ : the equilibrium dye concentration in the solution $\left(\mathrm{mg} . \mathrm{L}^{-1}\right)$; $\mathrm{q}_{\max }$ : the maximum adsorption capacity of the adsorbent $\left(\mathrm{mg} \cdot \mathrm{g}^{-1}\right) ; \mathrm{K}_{\mathrm{L}}$ : the Langmuir adsorption constant (L.mg ${ }^{-1}$ )

When the Freundlich isotherm model applies to adsorption on heterogeneous surfaces with interaction betweenthe adsorbed molecules, and is not restricted to the formation of a monolayer. This model assumes that asthe adsorbate concentration increases, the concentration of adsorbate on the adsorbent surface also increasesand, correspondingly, the sorption energy exponentially decreases. The wellknown expression for the Freundlich model is given by the linear equation (Eq.6):

$$
\operatorname{Logq}_{e}=\log K_{f}+\frac{1}{n_{F}} \cdot \log C_{e}
$$

Where

$\mathrm{q}_{\mathrm{e}}$ : the equilibrium dye concentration on the adsorbent (mg. $\left.\mathrm{g}^{-1}\right) ; \mathrm{C}_{\mathrm{e}}$ : the equilibrium dye concentration in the solution $\left(\mathrm{mg} . \mathrm{L}^{-1}\right) ; \mathrm{K}_{\mathrm{F}}$ : Proportionality constant for Freundlich equation $\left[\left(\mathrm{mg}^{-\mathrm{g}^{-1}}\right)\left(\mathrm{L} \cdot \mathrm{mg}^{-1}\right){ }^{1 / \mathrm{n}}\right] ; 1 / \mathrm{n}_{\mathrm{F}}$ : the adsorption intensity.

\section{RESULTS AND DISCUSSIONS}

\subsection{Effect of $\mathrm{pH}$ on adsorption process:}

In general, initial $\mathrm{pH}$ value may enhance or depress the uptake of solute. ThepH of adsorption medium influences not only the surface charge of adsorbent, but also, the degree of ionization of the material present in the solution and the dissociation of functional groups on the active sites of the adsorbent and the solution dye chemistry[5].The effect of $\mathrm{pH}$ on MBremoval was analyzed over the $\mathrm{pH}$ range from 2-12. The $\mathrm{pH}$ was adjusted using $0.1 \mathrm{~N}$ $(\mathrm{NaOH})$ or $0.1 \mathrm{~N}(\mathrm{HCl})$ solutions. In this work, $50 \mathrm{~mL}$ of dye solution was agitated with200mg of our biosorbentfor $120 \mathrm{~min}$ then the sample was centrifuged and analyzed using a spectrophotometer by measuring the absorbance changes at a wavelength of maximum absorbance $662 \mathrm{~nm}$. The Fig.3shows the effect of $\mathrm{pH}$ on Methylene Blue elimination, it's can be seen from the figure that the percentage removal of Methylene Blue by our biosorbent was optimum at basicpH $=10$, It could be as a result of the attraction between the positives charges of the cationic dye and the negatives of the sorbent. Hassan et al., (2013)[6]were obtained the similar basic adsorption $(\mathrm{pH}=8)$ of $\mathrm{MB}$ onto Haloxylonrecurvum plant stems

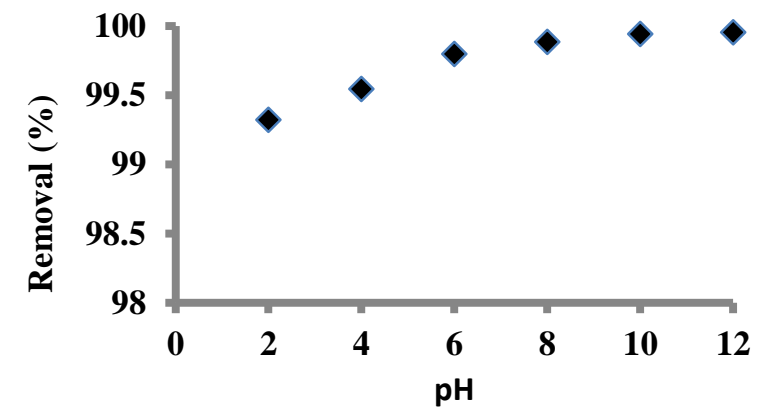




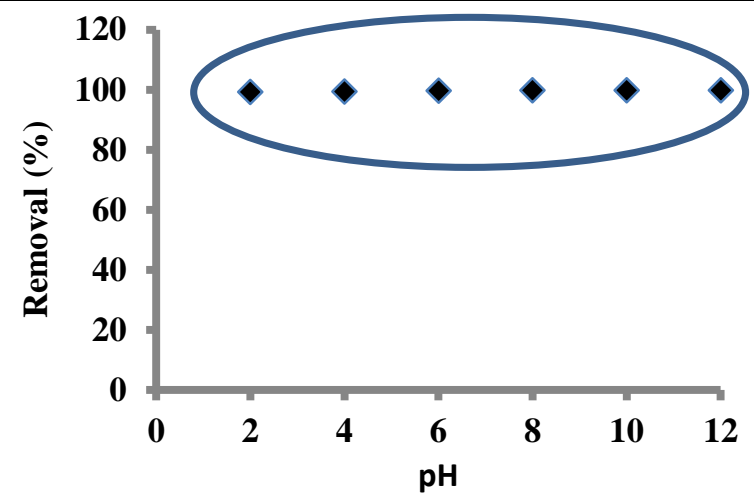

Fig. 1: Effect of $p H$ on Methylene Blue adsorption $(V=50 m L, t=2 H, m=200 \mathrm{mg}$ )

\subsection{Effect of adsorbent dose:}

Adsorbent dose is a very important factor that influences the sorption process. Thebiosorbent product at various doses was added to $50 \mathrm{~mL}$ of $\mathrm{MB}$ solution with initial concentration of $100 \mathrm{ppm}$ at optimal $\mathrm{pH}$ of 10 and atambient temperature.

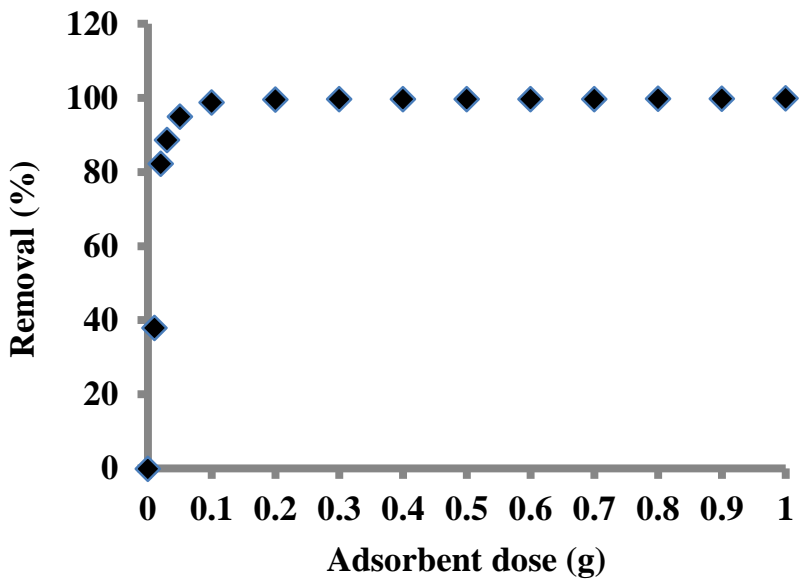

Fig.2: Effect of adsorbent dose on Methylene Blue adsorption $(\mathrm{V}=50 \mathrm{~mL}, \mathrm{t}=2 \mathrm{H}, \mathrm{pH}=10)$

The Fig.2revealed that the adsorption removal yield increase with the increasing of the adsorbent dose until the optimal value of adsorbent dose of $100 \mathrm{mg}$, after this value there was any significant change of the MB elimination.This can be explicated by the increases of the surface area and thus the number of available adsorption sites. Mahammedi and Belkacem (2015)[7]were obtained a maximum MB removal using $4 \mathrm{~g} / \mathrm{L}$ using natural clay.

\subsection{Effect of contact time and kinetic study:}

The plot of removal yield versus time is shown in Fig. 3.In this plot, it is apparent that MB removal by the adsorption increased rapidly in the initial stage (20 $\mathrm{min}$ ) and became slower in the later stages until the attainment of equilibrium.

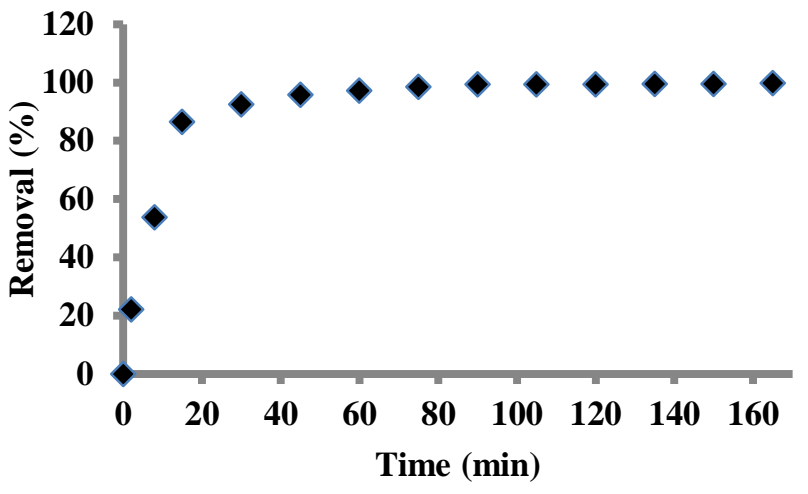

Fig.3: Effect of contact time on Methylene Blue adsorption $(V=500 m L, p H=10, m=1 g)$

As the surface adsorption sites become exhausted, the uptake rate is controlled by the rate at which the dye molecules are transported from the exterior to the interior sites of the adsorbent particles. Equilibrium time for the adsorption of MB found by Amuda et al.,(2014)[8]was found to be $60 \mathrm{~min}$ to reachonly an adsorption yield of 90\% using Steam-Activated Carbon Produced from Lantana camara Stem.

\subsubsection{Kinetic study:}

The plot of $t / q_{t}$ and $\operatorname{Ln}\left(\mathrm{q}_{\mathrm{e}}-\mathrm{q}_{\mathrm{t}}\right)$ versus time $\mathrm{t}$, were shown in the Fig.4

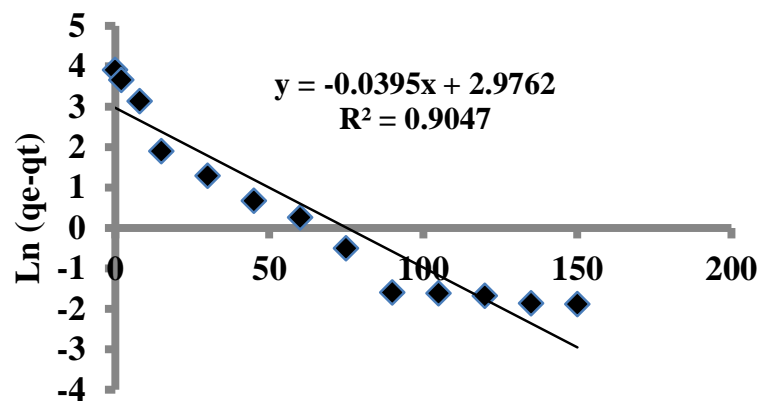

(a)

$\mathbf{t}(\min )$

Fig.4: (a) Pseudo first order,

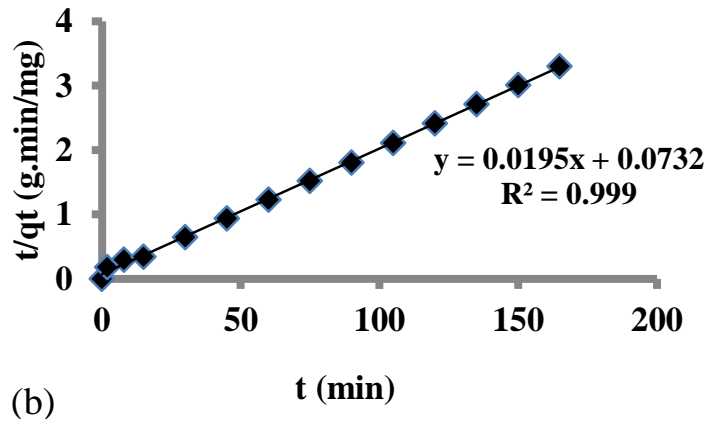

Fig.4: (b) Pseudo second order plot of papaya seed biosorbent 
From the results we observed that the adsorption of Methylene Blue was better fitted with the pseudo second order with a $\mathrm{R}^{2}=0.99$ (Table.1).

Table.1: Adsorption kinetic parameters of Methylene Blue

\begin{tabular}{ccccc}
\hline \multicolumn{2}{c}{ Pseudo-second order } & \multicolumn{3}{c}{ Pseudo first order } \\
\hline $\begin{array}{c}\text { Qe } \\
(\mathbf{m g} / \mathbf{g})\end{array}$ & $\begin{array}{c}\mathbf{K}_{\mathbf{2}} \\
(\mathbf{g} / \mathbf{m g} \text {. } \mathbf{m i n})\end{array}$ & $\mathbf{R}^{\mathbf{2}}$ & $\begin{array}{c}\mathbf{K}_{\mathbf{1}} \\
\left(\mathbf{m i n}^{-\mathbf{1}}\right)\end{array}$ & $\mathbf{R}^{\mathbf{2}}$ \\
\hline 51.28 & 0.133 & 0.99 & 0.039 & 0.90 \\
\hline
\end{tabular}

These results indicate that the kinetic model of the adsorption is based on the assumption that the rate-limiting step is a chemical adsorption involving valance force through sharing or exchange of electrons between adsorbent and adsorbate.Similar result was also obtained by Han et al., (2017) using Molybdenum Disulfide Nanostructure [9].

\subsubsection{Adsorption isotherm study:}

The adsorption capacity of the adsorbent, interaction between the solute-solution and the nature of adsorbed accumulation materials on the surface of the adsorbent can be explained using isotherm models. The Fig. 5 shows the plot of Langmuir and Freundlich isotherm models.

Results shown in Fig.5revealed that the biosorption of MB on papaya seed adsorbent is the monolayer type since the correlation coefficient $\left(\mathrm{R}^{2}\right)$ calculated from Langmuir isotherm is below 0.99 , which indicate lessapplicability for Langmuir isotherm.
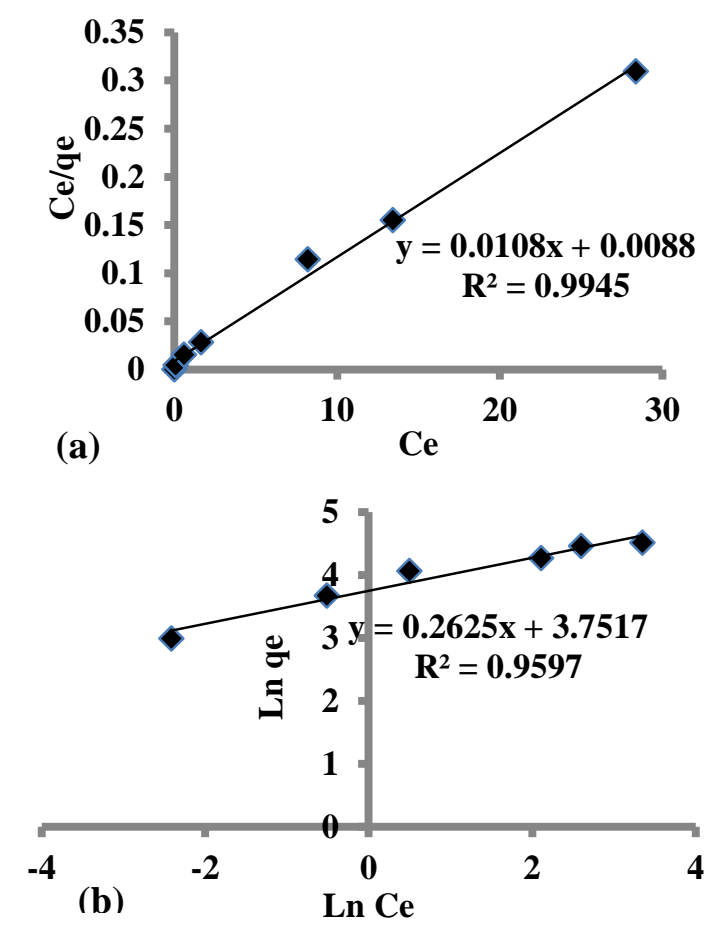

Fig.5 Adsorption isotherm of MB on papaya seed (a) Langmuir model, (b) Freundlich model
These results are in accordance with the result obtained in our Patent number PCT/MA2016/000003 for the removal of $\mathrm{MB}$ by combination of adsorption process into clay material and flocculation by polyelectrolyte extracted from cactus cladode.

\section{CONCLUSION}

Papaya seed were selected as a suitable agriculture product thanks its abundance in Morroco and its disposal in the environment without any treatment. Several parameters were studied to deduce their effect on Methylene Blue adsorption. The adsorption of Methylene Blue increase with the increasing of time, temperature and adsorbent dose. The results show that the optimal condition of treatment of $\mathrm{pH}=10$, adsorbent dose of $200 \mathrm{mg}$ in $100 \mathrm{~mL}$ and very low contact time of $20 \mathrm{~min}$ are sufficient to eliminate $98.8 \%$ of Methylene Blue. The adsorption isotherm was well reproduced with Langmuir model and show that the adsorption is the monolayer type, the kinetic study shows that the adsorption follows the pseudo second order model.

\section{REFERENCES}

[1] J. Lubchenco, Entering the century of the environment: a new social contract for science, Sci. 279(1998) 491-497.

[2] R. Srivastava, D. C. Rupainwar. A comparative evaluation for adsorption of dye on Neem bark and Mango bark powder. Indian J ChemTechnol(2011), 18: $67-75$

[3] M. Belbahloul, A. Anouar, A. Zouhri. Low Technology Water Treatment: Investigation of the Performance of Cactus Extracts as a Natural Flocculant for Flocculation of Local Clay Suspensions. International Journal of Engineering Research \& Technology.3 (3) (2014).

[4] S. Azizian, Kinetic models of sorption: a theoretical analysis, J. 32 Colloid Inter. Sci. 276 (2004) 47-52.

[5] M.S.Onyango, Y.Kojima, O.Aoyi, E.C.Bernardo, H.Mustafa, Adsorption equilibrium modeling and solution chemistry dependence of fluoride removal from water by trivalent cation-exchange zeolite F9, J. Colloid. Interface Sci.279 (2004) 341350

[6] W. Hassan. U. Farooq, M. Ahmad, M. A. Athar. Khan. Potential biosorbent, Haloxylonrecurvum plant stems, for the removal of methylene blue dye .Ar.J. Chem. 10 (2017) 1512-1522

[7] F. Mahammedi, B. Benguella. Adsorption of methylene blue from aqueous solutions using natural clay.J. Mater. Environ. Sci. 7 (1) (2016) 285-292

[8] O. Amuda, A. Olayiwola,A. Alade, A. Farombi, S. Adebisi.Adsorption of Methylene Blue from 
Aqueous Solution Using Steam-Activated Carbon

Produced from Lantana camara Stem. Journal of

Environmental Protection, 5(2014), 1352-1363.

[9] S. Han, K. Liu, L. Hu, F. Teng, P. Yu, Y. Zhu Superior Adsorption and Regenerable Dye Adsorbent Based on Flower-Like Molybdenum Disulfide Nanostructure. 2017. 